\title{
UNA INVITACIÓN A SEGUIR PENSANDO SOBRE LOS DERECHOS HUMANOS
}

\author{
Silvina Álvarez \\ Universidad Autónoma de Madrid
}

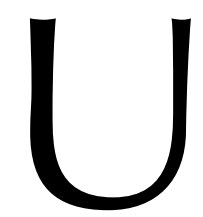

na de las importantes contribuciones de L. HIERRO y F. LAPORTA ha sido en el ámbito de los derechos humanos. Ambos se han ocupado del concepto, de su justificación, del contenido y de la titularidad de estos derechos, así como de los procesos históricos que contribuyeron a fraguar la idea; y su trabajo ha sido para mí un estímulo, una motivación, una invitación a seguir pensando sobre los derechos humanos. A partir de sus escritos sobre estas cuestiones, propongo aquí una reflexión sobre las posibilidades de una perspectiva que sin apartarse de los logros conseguidos en el ámbito del concepto y la justificación, nos permita avanzar hacia una noción más incluyente de los derechos humanos.

La teoría de los derechos humanos ha prestado en los últimos años, a través de diversos autores, una mayor atención a las prácticas sociales y políticas que intervienen en la configuración e institucionalización de los derechos. La propuesta apunta a tomar en cuenta el papel que juegan los derechos humanos en la vida política y jurídica, para dotar de eficacia las reivindicaciones que entrañan ${ }^{1}$. Siguiendo esta estela creo que resulta importante poner en relación las herramientas jurídicas con los sujetos a los que ellas se refieren, sus rasgos relevantes, su ámbito de relación y su contexto de actuación. Así, señalaré en lo que sigue la necesidad de revisar la perspectiva desde la que se presentan los derechos humanos de las mujeres, y de hacerlo pensando en el lugar desde el cual las mujeres plantean sus demandas de protección jurídica. A menudo se ha optado por un patrón o molde común, que sería válido para todos, por un lado, y desarrollos específicos, válidos para algunos titulares, entre ellos las mujeres ${ }^{2}$. Señalaré que esta forma de aproximación a los derechos humanos de las mujeres tergiversa a veces el enfoque y, en consecuencia, equivoca la caracterización de los mismos. Me inclinaré por la necesidad de una propuesta de actualización del contenido y la protección de los derechos humanos de las mujeres, lo cual no implica un nuevo modelo de los derechos humanos.

Aunque repensar el contenido de los derechos a menudo puede llevarnos a repensar algunos de los elementos del concepto, no creo que este ejercicio tenga que ponernos en la tesitura de abandonar el concepto original sino, más bien, de redimen-

1 Sobre este punto vid. J. MonTERo, 2016: 285-288.

2 Sobre «la tradición democrática de la especificación», vid. S. MECKLED-GARCíA, 2015: 301. 
sionar su alcance. Desde una perspectiva liberal-igualitaria, a menudo se entendió que la forma de corregir las distorsiones que el derecho podía reflejar en relación con los intereses específicamente femeninos, podía provenir de un tratamiento diferente para los casos que revelaban diferencias entre varones y mujeres. Sin embargo, a menudo esta concepción solo ha servido para plantear excepciones al modelo original, evitando una reflexión más amplia sobre el tipo de soluciones jurídicas adecuadas para casos singularmente distintos. En lo que sigue haré una breve referencia a la teoría relacional, marco desde el cual volver a pensar los derechos humanos.

\section{LOS DERECHOS DESDE LA PERSPECTIVA RELACIONAL}

HIERRO ha identificado los derechos humanos con «intereses o necesidades básicas» (1982) y LAPORTA se ha referido a su carácter absoluto en relación con la importancia de los bienes que protegen (1987: 37). Para lograr una noción de bien, necesidad o interés que sea receptiva a las demandas — genuinas y normativamente plausibles- de los titulares de derechos, resulta fundamental la aproximación a los sujetos-titulares. Este punto es importante porque a veces la construcción jurídica se presenta como el producto de un razonamiento imparcial que expresa sus enunciados sin atender a peculiaridades de ningún tipo, incluidas las del género. Esta perspectiva ha hecho que ese ideal, ese modelo abstracto o abstraído de las características reales de los sujetos, no tomara en cuenta rasgos constitutivos de los individuos-mujeres, rasgos sin los cuales no son tales individuos. La investigación jurídica feminista se ha ocupado de ver qué efectos producen normas pensadas en términos neutrales $-\mathrm{o}$ aparentemente neutrales- en relación con el género, cuando se aplican a situaciones de la vida - familiar, laboral, reproductiva, política, sexual - en las que ser varón o ser mujer ubica a las personas en escenarios de significado diferentes. Como señala Katharine BARTLETT en un conocido trabajo sobre metodología jurídica feminista, se puede rastrear una línea de trabajo feminista que no ha cesado de plantear «la pregunta sobre las mujeres» ${ }^{3}$, es decir, sobre las consecuencias que las normas, las prácticas consuetudinarias, las decisiones judiciales, las elaboraciones doctrinales, tienen para las mujeres.

En resumen, la propuesta es volver a pensar cuáles son los términos de lo universalizable teniendo en cuenta en quiénes recae la titularidad de los derechos ${ }^{4}$. En una mirada retrospectiva, vemos que la realización de la igualdad fue planteando la necesidad de reconocer no solo las semejanzas sino también las diferencias entre varones y mujeres, con vistas a alcanzar una tutela efectiva de la libertad. Como señala HiERRO, en las distintas fases de consolidación de los derechos humanos, se fueron asentando «derechos específicos de la mujer» (2016: 97). Con el tiempo, surgieron disposiciones legales específicas, como respuesta a situaciones singularmente femeninas. En el ámbito del trabajo, por ejemplo, se hizo necesario introducir salvaguardias con el objeto de

3 Sobre la metodología jurídica feminista y «the woman question», vid. BARLETT, 1991.

4 Sobre feminismo y universalismo, vid. Mullally, 2006: xxxii-xxxiii. Como afirma Niamh ReILly, la actualización de los derechos de las mujeres puede hacerse en la línea de autoras que reivindican el compromiso normativo con la universalidad, tales como OKIN, PHILLIPS, NussBAUM, entre otras (2009: 7). Sobre abstracción e idealización en relación con la propuesta de O. O’NeIll, vid. Beltrén, 2001: 194. Sobre la abstracción y la pretensión de neutralidad de las leyes, vid. también AÑón y MESTRE, 2005: 46-49. 
intentar impedir que el embarazo y la maternidad fueran causas de menoscabo en la vida laboral de las mujeres. En el ámbito penal, se fueron incorporando tipos penales que recogieran la especificidad de las agresiones perpetradas contra las mujeres, como la violación, el acoso sexual o la violencia de género.

Esta perspectiva tiene en cuenta como titular de derechos a un sujeto que carece de las llamadas «especificidades» y, de este modo, no llega a percibir que las características que encierra esa especificidad, no son apéndices o agregados al modelo inicial, sino notas centrales de sus titulares, sin las cuales difícilmente se pueda entender el tipo de protección jurídica que demandan. Lo específico forma parte, en realidad, de aquello que está en la base de la titularidad y, por tanto, convendría incorporarlo a la formulación original.

Jennifer NEDELSKY retoma estos temas para analizarlos desde la perspectiva relacional. Para lograr un derecho más incluyente, que recoja los aspectos que la singularidad de las mujeres presenta en la realidad (en relación con la reproducción, la sexualidad, la maternidad, la vida laboral y familiar, las relaciones de pareja, la integridad física, la conciliación, la dependencia), debemos partir de una reflexión sobre la igualdad moral y cómo el derecho puede contribuir a propiciarla. Desde esta perspectiva, tal vez la neutralidad deje de ser un valor central del sistema jurídico para pasar a ser un principio dependiente de la igualdad, cuya pertinencia deberá evaluarse en función de esta última. NEDELSKY propone pensar en los derechos a partir de las relaciones entre las personas, para poner en el centro del análisis el tipo de valores que las normas jurídicas pueden promover (2011: 65-68). Así, por ejemplo, en el derecho de familia, las leyes sobre el matrimonio promueven un tipo de relaciones íntimas al tiempo que desincentivan otros tipos de dichas relaciones (2011: 68), o en el derecho de obligaciones y contratos, las normas que regulan los derechos y obligaciones de arrendadores y arrendatarios han ido configurando, de manera progresiva a través de la historia, un escenario de mayores garantías y seguridad para los segundos (2011: 66).

De manera similar, la autora se detiene a analizar desde la perspectiva relacional, la configuración jurídica de la violencia entre varones y mujeres ${ }^{5}$. El enfoque de la autora parte de estudiar qué formas de intervención jurídica podrían propiciar un cambio de los patrones violentos de relación —antes que detenerse en el enfoque jurídico disuasorio que intenta solo frenar la violencia-. En este sentido, NEDELSKY propone pensar categorías nuevas tanto para el diagnóstico de la situación social sobre la que el derecho debe intervenir, como para diseñar instituciones jurídicas para la protección de las víctimas (2011: 204, 217). Por ejemplo, la autora estudia el consentimiento, noción nuclear del derecho penal para desentrañar la existencia de delitos contra la libertad sexual, a través de la legislación canadiense en la materia, que propone un estándar objetivo en contraposición con la construcción de la defensa basada en la creencia de que existía consentimiento (2011: 218-219). Conforme a dicha legislación, «no es eximente [...] que el acusado creyese que la demandante había consentido la actividad que constituye el objeto de los cargos formulados, cuando (a) la creencia del acusado haya surgido de (i) la intoxicación del acusado por él mismo inducida, o (ii) la falta de percepción de los

5 Siempre desde la perspectiva relacional, la autora se ocupa también de analizar cuestiones del ámbito constitucional, administrativo y otros. Vid. NEDELSKY, 2011. 
hechos por imprudencia o propia voluntad; o (b) el acusado no baya seguido los pasos razonables, en las circunstancias conocidas por el acusado en ese momento, para determinar que la demandante estaba consintiendo» ${ }^{6}$. NEDELSKY presenta esta legislación para ilustrar un tipo de construcción legal que incorpora la dimensión relacional, en la medida en que propugna a través de la configuración del delito de violación, una intervención jurídica atenta a los elementos contextuales que sirven de marco para construir las distintas opciones y posibilidades de acción, que desestima disposiciones negligentes o carentes de receptividad en las relaciones íntimas (2011: 220-221).

Otro ejemplo sobre el derecho y su capacidad para crear relaciones valorativamente comprometidas lo encontramos en las leyes que regulan la reproducción; estas marcan pautas y modelos de comportamiento, al tiempo que propician posiciones valorativas sobre ellos. En una materia como el aborto, tan sensible en numerosas sociedades, el derecho ha jugado un papel importante para reforzar estereotipos de mujer, de sexualidad y de maternidad; como afirma Robin WEST «necesitamos entender cómo las leyes que criminalizan el aborto construyen "la maternidad"» (2000: 169).

En relación con el embarazo como aspecto de la vida de las mujeres que requiere recepción jurídica, es interesante el itinerario de la jurisprudencia norteamericana en las primeras decisiones sobre subvenciones por embarazo, casos Geduldig v. Aiello (417 U.S. 484, 496-497, n. 20, 1974, equal protection) y General Electric Co. V. Gilbert (429 U.S. 125, 136-140, 1976, Title VII), en las que se negaba la universalidad de la prestación sanitaria por embarazo. El argumento entonces utilizado apelaba al hecho que el universo de quienes no se quedan embarazadas no es solo masculino, también hay mujeres que no se quedan embarazadas y, por tanto, no se trataría de una condición universalizable. En un caso posterior (California Federal Savings and Loan Association v. Guerra, 107 S. Ct. 683, 1987) en el que la Corte Suprema norteamericana concedió a una mujer embarazada una baja no pagada de cuatro meses, el razonamiento de la Corte consistió en centrarse en los costes que en términos de discriminación podía tener validar los acuerdos sociales existentes ${ }^{7}$.

Estos tortuosos desarrollos en torno al embarazo ponen de manifiesto las dificultades de los sistemas jurídicos para incorporar las peculiaridades de la titularidad femenina en la configuración tradicional de los derechos, y señalan la necesidad de revisitar los derechos humanos para intentar un contenido, una interpretación y una aplicación acordes con las demandas de intervención jurídica que plantea la posición de las mujeres en la sociedad.

\section{UNA REFLEXIÓN JURÍDICA ORIGINARIA}

Desde esta perspectiva, he querido apuntar la necesidad de pensar los derechos humanos de las mujeres teniendo en cuenta sus preocupaciones y contexto de relaciones ${ }^{8}$.

6 Canadian Criminal Code, R.S.C., 1985 chap. C-46, sec. 273.2, citado en Nedelsky, 2011: 219.

7 Vid. Minow, 1991: 364.

8 Carol Gould señala seis temas que aparecen de manera recurrente cada vez que se discuten los derechos humanos de las mujeres desde la perspectiva de esas preocupaciones, y que, según la autora, formarían el núcleo de las cuestiones a debatir: 1) las relaciones de responsabilidad y los vínculos de cuidado con otras per- 
Esta tarea nos aboca a lo que podríamos llamar una reflexión jurídica originaria. Esto no quiere decir que tengamos que prescindir ni de las bases de fundamentación que proporciona la concepción de los derechos humanos como derechos morales, ni de los elementos conceptuales generales que nos ayudan a definir el contenido de los derechos. Por el contrario, creo que la teoría moral, política y jurídica de los derechos humanos es un recurso indispensable para este ejercicio. Entiendo, en cambio, por «originaria» una reflexión no vinculada por concepciones jurídicas o constricciones dogmáticas que nos impidan hallar nuevas propuestas legales o interpretativas.

Tanto LAPORTA (2000) como HierRo (2016) han destacado cómo la historia de los derechos humanos se configura a través de la elaboración de conceptos fundamentales (derecho subjetivo, individualismo moral, autonomía, propiedad) que permitieron hacer frente a los abusos de poder. Como afirma HiERRO, «los derechos humanos se inventaron para proteger un ámbito de soberanía individual frente al poder físico o normativo de otros» (2016: 155). Si pensamos en la configuración de los primeros derechos humanos, los derechos civiles y políticos, nos encontramos con que en la génesis y evolución de estos derechos los individuos buscaron fórmulas para poder proteger sus espacios de libertad, salvaguardar su propiedad, realizar sus planes de vida, participar en el ámbito público, expresar sus ideas políticas, etc. Y en la búsqueda de las fórmulas para proteger su voluntad y a través de esta, sus intereses, se configuraron los derechos humanos de esta primera etapa como derechos contra el Soberano, ya que era precisamente del poder político de quien aquellos individuos querían protegerse?

Siguiendo esta pauta, podemos volver a pensar, sin constricciones, cómo se constituye el ámbito de soberanía individual de las mujeres, y frente a quiénes y con qué diseño jurídico se puede proteger mejor. Reflexionar sobre los ámbitos en los que las mujeres se sienten especialmente desprotegidas cuando tienen que ejercer su capacidad de elección, trazar su plan de vida o salvaguardar aquello que consideran valioso. Se trataría entonces de desvelar cuáles son las situaciones personales, sociales y culturales respecto de las cuales las mujeres se encuentran en una situación de mayor vulnerabilidad y, por tanto, demandan protección jurídica. Algunas de tales situaciones son ampliamente conocidas: el tráfico y trata de mujeres y niñas, la violencia sexual, el maltrato y la violencia doméstica, la prostitución, la pornografía, el acoso sexual, la violencia reproductiva, las esterilizaciones forzosas, los matrimonios forzosos, la mutilación genital, etc. Estos ejemplos vienen a señalar espacios de la vida de las mujeres — sexualidad, contexto doméstico, relaciones familiares, reproducción- en los que se hace especialmente necesaria la intervención del Estado a través de un sistema de derechos y garantías.

Una reflexión jurídica originaria sobre el contenido de los derechos humanos de las mujeres y sobre los medios adecuados para su protección jurídica nos brinda a su vez la oportunidad de repensar el concepto desde la perspectiva relacional, confrontando voluntades e intereses. Como lo expresara Alda FACIO, las cuestiones que preocupan en relación con las mujeres y el derecho no solo tienen que ver con la discriminación

sonas; 2) la distinción público-privado; 3) la conexión fuerte entre derechos de las mujeres y derechos sociales y económicos; 4) la viabilidad de la noción de derechos específicos para las mujeres; 5) los desafíos de la diversidad cultural, y 6) la globalización y la regionalización en relación con los derechos de las mujeres (2002: 4-5).

9 Vid. H. CHARLESWORTH, 1994: 71. 
en la aplicación de las normas, sino que «se deben también a las leyes que no existen, a todas las instituciones que no se han creado» (1999: 108).

\section{BIBLIOGRAFÍA}

AÑón Roig, M. J., y Mestre i Mestre, R., 2005: «Violencia sobre las mujeres: discriminación, subordinación y Derecho», en J. BoIX ReIG y E. MARTínez GARCÍA (coords.), La nueva Ley contra la Violencia de Género, Madrid: Iustel.

Bartlett, K. T., 1991: «Feminist Legal Methods», en K. Bartlett y R. Kennedy (eds.), Feminist Legal Theory. Readings in Law and Gender, Boulder: Westview Press.

BELTRÁN, E., 2001: «Justicia, democracia y ciudadanía: las vías hacia la igualdad», en E. BELTRÁN y V. MaquieIra, Feminismos. Debates teóricos contemporáneos, Madrid: Alianza.

CHARLESWORTH, H., 1994: «What are 'Women's International Human Rights'?», en R. J. COOK (ed.), Human Rights of Women. National and International Perspectives, Philadelpia: University of Pennsylvania Press.

FACIO, A., y Fries, L. (eds.), 1999: Género y Derecho, La Morada, Santiago de Chile: American University, Lom Ediciones.

Gould, C. C., 2002: «Conceptualizing Women's Human Rights», Working Paper, RSC núm. 2002/40, San Domenico, Firenze: European University Institute, Badia Fiesolana. También en C. C., Globalizing Democracy and Human Rights, Cambridge University Press, 2004, 139-155.

Hierro, L., 1982: «Derechos humanos o necesidades humanos. Problemas de un concepto», Sistema, núm. 46.

- 2016: Los derechos humanos. Una concepción de la justicia, Madrid: Marcial Pons.

LAPORTA, F., 1987: «Sobre el concepto de derechos humanos», Doxa, núm. 4.

- 2000: «El concepto de los derechos humanos», en R. Soriano Díaz, C. Alarcón Cabrera y J. J. Mora Molina (eds.), Diccionario Crítico de los Derechos Humanos, Universidad Internacional de Andalucía.

Meckled-García, S., 2015: «Specifying Human Rights», en Cruft, LiaO y Renzo (eds.), Philosophical Foundations of Human Rights, Oxford University Press.

Minow, M., 1991: «Feminist Reason: Getting It and Losing It», en K. T. BARTLETT y R. KeNNEDY (eds.), Feminist Legal Theory. Readings in Law and Gender, Boulder: Westview Press.

MONTERO, J., 2016: «Una metodología para pensar sobre los derechos humanos», en G. LARIGUET (comp.), Metodología de la investigación jurídica, Argentina: Brujas.

Mullally, S., 2006: Gender, Culture and Human Rights. Reclaiming Universalism, Oxford: Hart Publishing.

Nedelsky, J., 2011: Law's Relations. A Relational Theory of Self, Autonomy, and Law, Oxford University Press.

ReILly, N., 2009: Women's Human Rights. Seeking Gender Justice in a Globalizing Age, Cambridge, UK: Polity Press.

West, R., 1988: «Jurisprudence and Gender», University of Chicago Law Review, vol. 55, núm. 1, 1-70. Traducción al castellano de P. LAMA LAMA, Género y teoría del derecho, Bogotá: Ediciones Uniandes. 